\title{
On the Performance of the DNPS-Based Relay Networks under Masquerading Attack
}

\author{
Wenson Chang (iD) \\ Department of Electrical Engineering, National Cheng Kung University, Tainan 701, Taiwan \\ Correspondence should be addressed to Wenson Chang; wenson@ee.ncku.edu.tw
}

Received 8 March 2018; Revised 15 June 2018; Accepted 2 July 2018; Published 17 July 2018

Academic Editor: Yafei Hou

Copyright (C) 2018 Wenson Chang. This is an open access article distributed under the Creative Commons Attribution License, which permits unrestricted use, distribution, and reproduction in any medium, provided the original work is properly cited.

\begin{abstract}
In the relay networks, two typical issues of physical layer security are selfishness and garbling. As a matter of fact, a certain nontypical but severely harmful misbehavior can also remove the cooperative diversity gain. Here, we coin the masquerading attack to indicate this kind of misbehavior. A masquerade relay can always pretend to be the best one to forward signals and, in consequence, deprive the others of the opportunities to cooperate. To the best of our knowledge, the impact of the masquerading attack has not yet been fully investigated. In this paper, multiple masquerade relays with random masquerading behavior are taken into account. Also, the complete channel effects, including the effects of the flat Rayleigh fading, log-normal shadowing, and path loss, are considered such that the geographical effects of the network topology can be completely captured. At last, the impact of the masquerade relays are evaluated in terms of the outage probability and end-to-end capacity.
\end{abstract}

\section{Introduction}

Nowadays, the hyperdense heterogeneous network (HetNet) has been widely recognized as a necessity to boost data rate for future generation of wireless communication systems [13]. With hyperdense deployment, the cooperative communication technologies can effectively extend system coverage and enhance quality of service (QoS). One important paradigm to accomplish these tasks is the cooperative relay networks [4-7]. In the literature, many aspects of the relay networks have been investigated, including the relay selection scheme, network code design, and power allocation. However, one important issue about the physical layer security in the relay networks is still not being completely inspected, i.e., the masquerading attack.

In the relay networks, two typical issues of physical layer security are the selfishness and garbling $[8,9]$. In the selfishness scenarios, the hypocritical relays may forward signals using minimum transmission power or, even worse, refuse to transmit any in-transit messages [10-12]. On the other hand, the in-transit messages may also be garbled [10, 13]. To unmask the hypocritical relays, some specific tracing symbols can be added to the informative messages $[10,13]$; otherwise, the malicious detection can also be conducted blindly, based on the characteristics of hybrid automatic repeat request [11], the credit-based incentive transmission scheme [12], or the received signal's correlation [14].

In addition to the selfishness and garbling, we find that a certain nontypical but severely harmful misbehavior can also deprive the relay networks of cooperative diversity gain. It is well-known that a cooperative relay can be opportunistically selected to forward signals based on the distributed network path selection (DNPS) protocol [15]. In DNPS, each candidate relay can set a timer according to channel gain; and the best cooperative relay can then be distributedly decided once its timer expires earlier than the others. However, in this scenario, it is highly possible that a hypocritical relay can maliciously set a timer which can always expire earliest, even though it owns the worst channel gain. Although the signals are forwarded, the degree of freedom (DoF) as well as the cooperative diversity gain can be seriously weakened [16]. As a result, the advantage of deploying the hyperdense relay networks can be seriously diluted. To clearly indicate this problem, we pioneeringly coin masquerading attack to describe this kind of misbehavior.

To the best of our knowledge, the masquerading attack has not yet been fully investigated. Although its impact has been analyzed in [16], only single masquerade relay 
was considered. Likewise, it neglected the complete channel effects; i.e., only the Rayleigh fading with different variances was included. In consequence, the geographical effects of the network topologies can not be completely characterized by the analytical results. Here, to capture the complete effects of fading environment, including the effects of flat Rayleigh fading, log-normal shadowing, and path loss, the composite exponential log-normal (CELN) distributed channel gain is considered [17]. Furthermore, multiple masquerade relays with random masquerading behavior are taken into account, i.e., the probability of a relay to become a masquerade relay and probability of a masquerade relay to become active. Then, the impact of the masquerading attack is evaluated in terms of the outage probability and end-to-end capacity. Note that part of this work has been presented in IEEE Wireless Communications and Networking Conference 2017 [18]. However, herein, some important related works are surveyed, and all the details of the mathematical derivations are provided. Furthermore, additional topologies of relay networks (as shown in Figures 2(b) and 5) are considered to investigate the influence of the masquerading attack on the device-to-device (D2D) and cellular networks.

The rest of this paper is organized as follows. In Section 2, the system model of the DNPS-based relay network is introduced. Also, the problem description is expounded therein. Section 3 mathematically describes the masquerading behaviors. Then, the outage probability and end-to-end capacity are derived in Section 4. Simulation results and conclusion remarks, including some suggestions for future works, are given in Sections 5 and 6, respectively.

\section{System Model}

Assume that $N$ relays are deployed to assist the data transmissions between the source $S$ and destination $D$. The decode-and-forward protocol is applied for relay-assisted transmissions. That is, during Phase I's transmission period, the $i$-th node $R_{i}$ can be included into the decodable set $\mathscr{D}(S)$ when its normalized capacity $C_{i}^{I}$ is larger than the predefined threshold $C_{t h}$ as

$$
C_{i}^{I}=\log _{2}\left(1+\left|h_{S R_{i}}\right|^{2} \gamma_{S}\right) \geq C_{t h}
$$

where $\gamma_{S}=P_{S} / N_{0}$ is the transmitting signal-to-noise ratio (SNR) at the source; $P_{S}$ is the source's transmission power; $N_{0}$ is the power spectrum density of the additive white Gaussian noise; $\left|h_{S R_{i}}\right|$ is the channel gain of the link between $S$ and $R_{i}$. To capture the complete effects of fading environment, including the effects of flat Rayleigh fading, log-normal shadowing and path loss, the CELN distributed channel gain are considered [17]. Thus, the probability density function $(p d f)$ of $\left|h_{S R_{i}}\right|^{2}$ can be modeled by

$$
f_{i}^{I}(\zeta)=\frac{\xi}{\sqrt{2 \pi} \hat{\sigma} \zeta} \exp \left[-\frac{\left(\xi \ln \zeta-\widehat{\mu}_{i}^{I}\right)^{2}}{2 \widehat{\sigma}^{2}}\right], \quad \zeta>0,
$$

where $\widehat{\mu}_{i}^{I}=\mu_{i}^{I}-\xi \kappa ; \widehat{\sigma}=\sqrt{\sigma+5.57^{2}}$; and $\kappa \approx 0.577$ is Euler's constant; $\mu_{i}^{I}$ and $\sigma$ are the mean and standard deviation (std.) of the log-normal shadowing in the $\mathrm{dB}$ domain during Phase I's transmission period; $\xi=10 / \ln 10$. Note that the mean of $\left|h_{S R_{i}}\right|^{2}$ is distance-dependent. Thus, a superscription is needed to distinguish $\mu_{i}^{I}$ and $\widehat{\mu}_{i}^{I}$ during Phase I from those during Phase II, i.e., $\mu_{i}^{I I}$ and $\widehat{\mu}_{i}^{I I}$. Specifically, the $\mu_{i}^{I I}$ and $\hat{\mu}_{i}^{I I}$ are associated with $\left|h_{R_{i} D}\right|^{2}$, where $h_{R_{i} D}$ stands for the channel gain of the link between $R_{i}$ and $D$. Since only one hop is required for the transmissions via the direct link, no superscription is needed for $\mu_{d}$ and $\widehat{\mu}_{d}$ (which are associated with $\left|h_{S D}\right|^{2}$ ). Similarly, there is no superscription to distinguish $\widehat{\sigma}$ and $\sigma$ during Phase I from those during Phase II because the same environment is assumed for both Phases I and II. Moreover, the cumulative distribution function $(c d f)$ of the CELN distribution can be obtained by integrating (2) as follows:

$$
F_{i}^{I}(\zeta)=\int_{0}^{\zeta} f_{i}^{I}(\eta) d \eta=Q\left(\frac{\hat{\mu}_{i}^{I}-\xi \ln \zeta}{\widehat{\sigma}}\right) .
$$

During Phase II's transmission period, one of the candidate nodes in $\mathscr{D}(S)$ is selected to forward data packets to the destination $D$ using the DNPS protocol [15] (will be briefly introduced in the latter). Finally, at the destination, the signal directly from $S$ and that from the selected relay are combined according to the maximal ratio combining (MRC) rule, which gives the effective end-to-end capacity as

$$
C_{i}^{I I}=\frac{1}{2} \log _{2}\left(1+\left|h_{S D}\right|^{2} \gamma_{S}+\left|h_{R_{i} D}\right|^{2} \gamma_{R}\right),
$$

where $\gamma_{R}=P_{R} / N_{0}$ is the transmitting SNR at the relay; $P_{R}$ is relay's transmission power; $h_{S D}$ represents the channel gain of the direct link. Note that when $\mathscr{D}(S)$ is empty, only the signal received via the direct link, i.e., from $S$ to $D$, is used for the demodulation process. In this case, the end-to-end capacity $C_{S}$ can be expressed as

$$
C_{S}=\log _{2}\left(1+\left|h_{S D}\right|^{2} \gamma_{S}\right)
$$

2.1. DNPS Relay Selection. Generally speaking, the DNPS protocol is an efficient distributed algorithm for relay selection. In the DNPS protocol, each candidate relay $R_{i}$ belonging to $\mathscr{D}(S)$ sets a timer $T_{i}$ whose expiry period is set inversely proportional to its channel gain of the link towards the destination $D$, i.e., $\left|h_{R_{i} D}\right| \forall i=1, \ldots, N$. In other words, a relay with the largest channel gain in Phase II can expire earliest. Once a timer expires, the associated relay broadcasts a flag signal to inform the neighboring relays so that it can solely occupy the channel for delivering packets in Phase II. More details about the DNPS protocol can be found in [15].

2.2. Problem Description. To begin with, the "masquerader" and "nonmasquerader" are defined as the "masquerade" and "ordinary" relays, respectively. Now, it is assumed that the masqueraders attack the relay-assisted networks by mimicking virus' behavior so that it can be violent and untraceable. Specifically, it can be contagious; and the infected relays can be asymptomatic carrier or explicitly symptomatic. To well describe this kind of masquerading attack, $P_{\alpha}$ is 


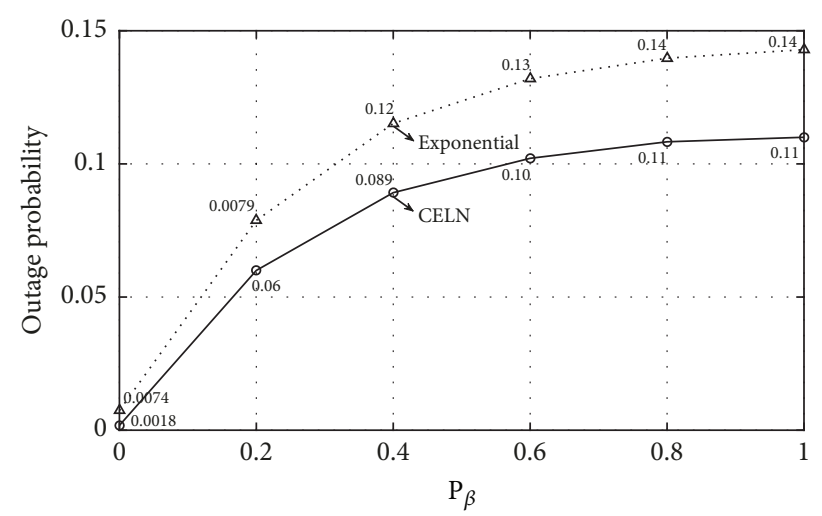

(a) Outage probability

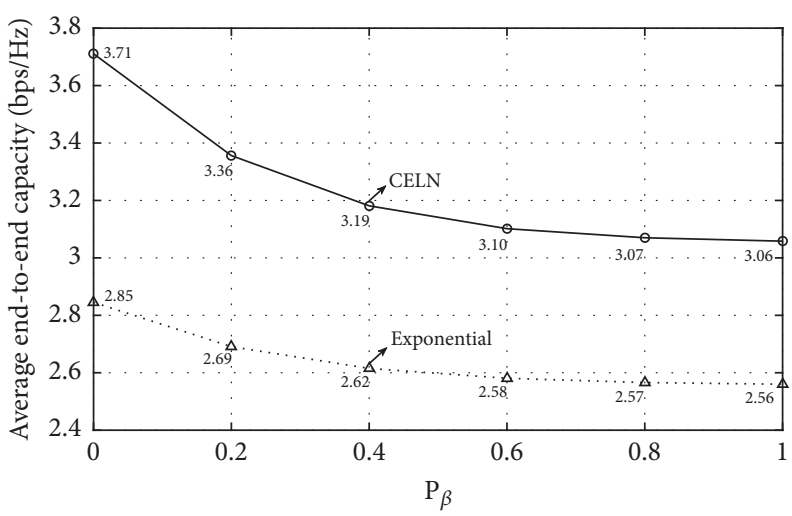

(b) Average end-to-end capacity

FIGURE 1: (a) Outage probability and (b) average end-to-end capacity with respect to $P_{\beta}$ for the infected relay-assisted cellular network under the exponential and CELN channel environments, where $P_{\alpha}=0.5$. Also, $N=9$ relays are fixed at middles of the sector as illustrated in Figure 5(a). Therein, the radius of the cell is $R_{\text {cell }}=1000 \mathrm{~m}$, and the location of the mobile station (MS) is uniformly distributed over the sector's coverage area, while the minimum distance between the MS and base-station (BS) is $50 \mathrm{~m}$. The transmission power of the source (i.e., the MS) $P_{S}$ is set so that the thermal noise outage $\left(O_{N}\right)$ at the destination (i.e., the BS) can be 0.2 as the MS is at the cell edge [19]. Similarly, the transmission power of each relay $P_{R}$ is set so that $O_{N}=0.2$ can be achieved at the BS. The required SNR corresponding to $O_{N}=0.2$ is defined as $0 \mathrm{~dB}$, whereas, for the purpose of evaluating the outage probability, the SNR threshold is set at $8 \mathrm{~dB}$. The results are obtained by averaging over 200,000 simulation rounds.

defined as the probability for an ordinary relay to become a masquerader. Moreover, a masquerader can be active (i.e., explicitly symptomatic in other words) and attack with probability $P_{\beta}$. Note that this kind of masquerading attack was ignored in the conventional counterpart [16]. Moreover, solely the Rayleigh fading with different variances (i.e., the exponential channel model) was considered therein. Figure 1 demonstrates the performance degradation for the infected relay-assisted cellular network under the exponential and CELN channel environments. Apparently, the masquerading attack can cause serious performance degradation. Moreover, the performance differences between the cases under the exponential and CELN channel environments are significant. Thus, one can tell that it is necessary and important to investigate the impact of the random masquerading attack by taking the CELN environment into account. Note that the larger dynamic range of the channel gain incurred by log-normal shadowing results in the higher diversity gain, which explains the better performance for the CELN environment.

\section{Analytical Characteristics of Masquerader}

The impact of masquerading attack will be evaluated in terms of the outage probability and end-to-end capacity in Section 4. To this end, two scenarios of the decodable set $\mathscr{D}(S)$ are firstly analyzed in this section, i.e., at least one masquerader in $\mathscr{D}(S)$ and no masqueraders in the nonempty $\mathscr{D}(S)$.

3.1. At Least One Masquerader in $\mathscr{D}(S)$. In this scenario, the impact of the ordinary relays belonging to $\mathscr{D}(S)$ can be ignored. This is because once an active masquerader exists in the decodable set $\mathscr{D}(S)$, the ordinary relays can never be selected to forward packets during Phase II. To facilitate the presentation, some terminologies are defined as follows.

(1) $\mathbf{M}(n)$ : the relay set which includes all possible combinations (subsets in other words) of $n$ masqueraders, where $1 \leq n \leq N$. Specifically, that means $n$ out of the $N$ relays belonging to $\mathbf{N}$ become masqueraders. Therefore, in this case, there are $\left(\begin{array}{l}N \\ n\end{array}\right)$ subsets in $\mathbf{M}(n)$, which are denoted by $\mathbf{M}(n, i) \forall i=1, \ldots,\left(\begin{array}{l}N \\ n\end{array}\right)$.

(2) $\mathbf{M}_{d}(m)$ : under the condition of $n$ masqueraders, this relay set includes all possible combinations of $m$ decodable masqueraders, where $1 \leq m \leq n \leq N$. In other words, $m$ out of the $n$ masqueraders belonging to $\mathbf{M}(n, i)$ are decodable. Therefore, given $\mathbf{M}(n, i)$, there are $\left(\begin{array}{c}n \\ m\end{array}\right)$ subsets in $\mathbf{M}_{d}(m)$, which are denoted by $\mathbf{M}_{d}(m, j) \subseteq \mathbf{M}(n, i), \forall j=1, \ldots,\left(\begin{array}{c}n \\ m\end{array}\right)$.

(3) $\mathbf{M}_{a}(\ell)$ : under the conditions of $n$ masqueraders and $m$ decodable masqueraders, this relay set includes all possible combinations of $\ell$ active-and-decodable masqueraders, where $1 \leq \ell \leq m \leq n \leq N$. That means $\ell$ out of the $m$ masqueraders belonging to $\mathbf{M}_{d}(m, j)$ are decodable. In this case, there are $\left(\begin{array}{c}m \\ \ell\end{array}\right)$ subsets in $\mathbf{M}_{a}(\ell)$, which are denoted by $\mathbf{M}_{a}(\ell, k) \subseteq \mathbf{M}_{d}(m, j) \subseteq$ $\mathbf{M}(n, i), \forall k=1, \ldots,\left(\begin{array}{c}m \\ \ell\end{array}\right)$.

Consider a masquerader being selected from a particular subset $\mathbf{M}_{a}(\ell, k)$. Since $\mathbf{M}_{a}(\ell, k) \subseteq \mathbf{M}_{d}(m, j) \subseteq \mathbf{M}(n, i)$, the joint probability for this case can be expressed as (6).

$$
\begin{aligned}
P & {\left[\mathbf{M}_{a}(\ell, k), \mathbf{M}_{d}(m, j), \mathbf{M}(n, i)\right] } \\
& =P\left[\mathbf{M}_{a}(\ell, k) \mid \mathbf{M}_{d}(m, j), \mathbf{M}(n, i)\right] \\
& \cdot P\left[\mathbf{M}_{d}(m, j) \mid \mathbf{M}(n, i)\right] P[\mathbf{M}(n, i)]
\end{aligned}
$$


Apparently, we can have

$$
P[\mathbf{M}(n, i)]=\left(P_{\alpha}\right)^{n}\left(1-P_{\alpha}\right)^{N-n} .
$$

Also, the conditional probability $P\left[\mathbf{M}_{d}(m, j) \mid \mathbf{M}(n, i)\right]$ can be expressed as

$$
\begin{aligned}
& P\left[\mathbf{M}_{d}(m, j) \mid \mathbf{M}(n, i)\right] \\
& =\prod_{k \in \mathbf{M}_{d}(m, j)}^{m} P\left[C_{k}^{I}>C_{t h}\right] \\
& \quad \times \prod_{k \in\left(\mathbf{M}(n, i) / \mathbf{M}_{d}(m, j)\right)}^{n-m} P\left[C_{k}^{I} \leq C_{t h}\right],
\end{aligned}
$$

where $\mathbf{A} / \mathbf{B}$ is the set-operator to remove set $\mathbf{B}$ from set $\mathbf{A}$;

$$
\begin{aligned}
P\left[C_{k}^{I} \leq C_{t h}\right] & =P\left[\left|h_{S R_{k}}\right|^{2}<\frac{2^{C_{t h}}-1}{\gamma_{S}}\right] \\
& =Q\left(\frac{\widehat{\mu}_{k}^{I}-\xi \ln \lambda_{t h}}{\widehat{\sigma}}\right),
\end{aligned}
$$

where $\lambda_{t h}=\left(2^{C_{t h}}-1\right) / \gamma_{S}$. Since

$$
Q\left(\frac{\xi \ln \lambda_{t h}-\widehat{\mu}_{k}^{I}}{\widehat{\sigma}}\right)+Q\left(\frac{\widehat{\mu}_{k}^{I}-\xi \ln \lambda_{t h}}{\widehat{\sigma}}\right)=1,
$$

we can have

$$
P\left[C_{k}^{I}>C_{t h}\right]=Q\left(\frac{\xi \ln \lambda_{t h}-\widehat{\mu}_{k}^{I}}{\widehat{\sigma}}\right) .
$$

Moreover, it is intuitional to obtain

$$
\begin{aligned}
P & {\left[\mathbf{M}_{a}(\ell, k) \mid \mathbf{M}_{d}(m, j), \mathbf{M}(n, i)\right] } \\
& =\left(P_{\beta}\right)^{\ell}\left(1-P_{\beta}\right)^{(m-\ell)} .
\end{aligned}
$$

At last, multiplying (7), (8), and (12) gives (6). It should be noticed that, with $\ell \geq 2$, the following performance metric (i.e., the outage probability and capacity in Section 4) should be averaged over the $\ell$ cases. This is because each of the $\ell$ masquerade relays individually sets a timer such that it can expire earliest. Then, the relay selection in Phase II becomes a random selection approach, which means each of them can be selected with probability $1 / \ell$.

3.2. No Active Masqueraders in the Nonempty $\mathscr{D}(S)$. As implied by the name, this scenario means that all the relays belonging to $\mathscr{D}(S)$ are ordinary ones. Thus, it includes three cases, i.e., (1) Case $\mathscr{A}$ : no relays become the masqueraders; (2) Case $\mathscr{B}$ : no masqueraders are decodable; and (3) Case $\mathscr{C}$ : no decodable masqueraders are active. Some terminologies are defined as follows:

(1) $\mathbf{O}(n)$ : the relay set includes all possible combinations of $n$ nonmasqueraders, where $1 \leq n \leq N$. Thus, there are $\left(\begin{array}{l}N \\ n\end{array}\right)$ subsets in $\mathbf{O}(n)$, which are denoted by $\mathbf{O}(n, i) \forall i=1, \ldots,\left(\begin{array}{l}N \\ n\end{array}\right)$.
(2) $\mathbf{O}_{d}(m)$ : under the condition of $n$ nonmasqueraders, this relay set includes all possible combinations of $m$ decodable nonmasqueraders, where $1 \leq m \leq$ $n \leq N$. That means given $\mathbf{O}(n)$, there are $\left(\begin{array}{c}n \\ m\end{array}\right)$ subsets in $\mathbf{O}_{d}(m)$, which are denoted by $\mathbf{O}_{d}(m, i) \forall i=$ $1, \ldots,\left(\begin{array}{c}n \\ m\end{array}\right)$.

(3) $\overline{\mathbf{M}}_{d}(n, i)$ : the relay set consists of the same masqueraders as $\mathbf{M}(n, i)$. However, all of the $n$ masqueraders are nondecodable. By definition, we can have $\overline{\mathbf{M}}_{d}(n)=$ $\overline{\mathbf{M}}_{d}(n, 1) \cup \overline{\mathbf{M}}_{d}(n, 2) \cdots \cup \overline{\mathbf{M}}_{d}\left(n,\left(\begin{array}{c}N \\ n\end{array}\right)\right)$.

(4) $\overline{\mathbf{M}}_{a}(m, j)$ : the relay set consists of the same masqueraders as $\mathbf{M}_{d}(m, j)$. However, all the $m$ decodable masqueraders are inactive. Given $\mathbf{M}_{d}(n, i)$, we can have $\overline{\mathbf{M}}_{a}(m)=\overline{\mathbf{M}}_{a}(m, 1) \cup \overline{\mathbf{M}}_{a}(m, 2) \cdots \cup \overline{\mathbf{M}}_{a}\left(m,\left(\begin{array}{c}n \\ m\end{array}\right)\right)$.

(1) Case A: No Relays Become the Masqueraders. Firstly, all the relays are well-behaved with probability

$$
P[\mathbf{O}(N)]=\left(1-P_{\alpha}\right)^{N} ;
$$

and the conditional probability $P\left[\mathbf{O}_{d}(n, i) \mid \mathbf{O}(N)\right]$ can be obtained by replacing $\mathbf{M}_{d}(m, j), \mathbf{M}(n, i), n$, and $m$ in (8) with $\mathbf{O}_{d}(n, i), \mathbf{O}(N), N$, and $n$, respectively.

(2) Case $\mathscr{B}:$ No Masqueraders Are Decodable. Consider that $\overline{\mathbf{M}}_{d}(n, i)$ occurs on a given condition of $\mathbf{M}(n, i)$. The conditional probability $P\left[\overline{\mathbf{M}}_{d}(n, i) \mid \mathbf{M}(n, i)\right]$ can be written as

$$
\begin{aligned}
P & {\left[\overline{\mathbf{M}}_{d}(n, i) \mid \mathbf{M}(n, i)\right]=\prod_{k \in \overline{\mathbf{M}}_{d}(n, i)}^{n} Q\left(\frac{\widehat{\mu}_{k}^{I}-\xi \ln \lambda_{t h}}{\widehat{\sigma}}\right) } \\
& =\prod_{k \in \mathbf{M}(n, i)}^{n} Q\left(\frac{\widehat{\mu}_{k}^{I}-\xi \ln \lambda_{t h}}{\widehat{\sigma}}\right) .
\end{aligned}
$$

Assume that $m$ out of the rest $N-n$ nonmasqueraders belonging to $\mathbf{N} / \overline{\mathbf{M}}_{d}(n, i)$ are decodable. Let $\mathbf{O}_{d}(m, j)$ represent these $m$ decodable nonmasqueraders. Then, replacing $\mathbf{M}_{d}(m, j)$, $\mathbf{M}(n, i)$, and $n$ in (8) with $\mathbf{O}_{d}(m, j), \mathbf{N} / \overline{\mathbf{M}}_{d}(n, i)$, and $N-n$ gives $P\left[\mathbf{O}_{d}(m, j) \mid \overline{\mathbf{M}}_{d}(n, i), \mathbf{M}(n, i)\right]$.

(3) Case $\mathscr{C}$ : No Decodable Masqueraders Are Active. Consider that $\overline{\mathbf{M}}_{a}(m, j)$ occurs on the given conditions of $\mathbf{M}_{d}(m, j)$ and $\mathbf{M}(n, i)$. Then, it leads to the conditional probability as

$$
P\left[\overline{\mathbf{M}}_{a}(m, j) \mid \mathbf{M}_{d}(m, j), \mathbf{M}(n, i)\right]=\left(1-P_{\beta}\right)^{m} .
$$

Assume that $\ell$ out of the remaining $N-n$ nonmasqueraders belonging to $\mathbf{N} / \mathbf{M}(n, i)$ are decodable. Let $\mathbf{O}_{d}(\ell, k)$ represent these $\ell$ decodable nonmasqueraders; and then we can have the conditional probability $P\left[\mathbf{O}_{d}(\ell, k)\right.$ । $\left.\overline{\mathbf{M}}_{a}(m, j), \mathbf{M}_{d}(m, j), \mathbf{M}(n, i)\right]$ by replacing $\mathbf{M}_{d}(m, j), \mathbf{M}(n, i)$, $n$, and $m$ in (8) with $\mathbf{O}_{d}(\ell, k), \mathbf{N} / \mathbf{M}(n, i), N-n$, and $\ell$, respectively.

\section{Performance Analysis}

Let the $z$-th relay be selected to forward packets during Phase II. Therefore, $z$ must belong to a particular subset of $\mathbf{M}_{a}(\ell)$ or 
$\mathbf{O}_{d}(n)$, e.g., the aforementioned $\mathbf{M}_{a}(\ell, k), \mathbf{O}_{d}(n, i), \mathbf{O}_{d}(m, j)$, or $\mathbf{O}_{d}(\ell, k)$ in Section 3. To ease the presentation, let $x=$ $\left|h_{R_{z} D}\right|^{2}$; and then $\mu_{x}$ stands for the mean of $x$ in the $\mathrm{dB}$ domain, which leads to $\widehat{\mu}_{x}=\mu_{x}-\xi_{\kappa}$. Moreover, $F_{X}(x)$ and $f_{X}(x)$ represent the $c d f$ and $p d f$ of $x$, respectively. Similarly, let $y=\left|h_{S D}\right|^{2}$; and, in consequence, we can have $\mu_{y}$ to represent the mean of $y$ in the $\mathrm{dB}$ domain, which leads to $\widehat{\mu}_{y}=\mu_{y}-\xi \kappa$. Likewise, $F_{Y}(y)$ and $f_{Y}(y)$ stand for the $c d f$ and $p d f$ of $y$.

Denote $P_{\text {out }}(M)$ and $P_{\text {out }}(O)$ as the outage probability for the cases of $z$ belonging to a subset of $\mathbf{M}_{a}(\ell)$ or $\mathbf{O}_{d}(n)$, respectively, while $P_{\text {out }}(\emptyset)$ denotes that with an empty decodable set $\mathscr{D}(S)$. Also, let $C_{\text {end }}(M), C_{\text {end }}(O)$, and $C_{\text {end }}(\emptyset)$ represent the corresponding average end-to-end capacity. Since $P_{\text {out }}(M)$, $P_{\text {out }}(O)$, and $P_{\text {out }}(\emptyset)$ are mutually exclusive, the overall outage probability can be expressed as

$$
P_{\text {out }}=P_{\text {out }}(M)+P_{\text {out }}(O)+P_{\text {out }}(\emptyset) .
$$

Similarly, we can have

$$
C_{\text {end }}=C_{\text {end }}(M)+C_{\text {end }}(O)+C_{\text {end }}(\emptyset) .
$$

In the following, we derive the mathematical expressions of $P_{\text {out }}(M), P_{\text {out }}(O)$, and $P_{\text {out }}(\emptyset)$. The closed form expressions of $C_{\text {end }}(M), C_{\text {end }}(O)$, and $C_{\text {end }}(\emptyset)$ will be derived as well.

4.1. At Least One Masquerader in $\mathscr{D}(S)$. To facilitate the presentation, denote $P_{M}(z \mid \ell, m, n, k, j, i)$ as the probability of the selected $z$-th relay belonging to the subset $\mathbf{M}_{a}(\ell, k)$. Then, it gives

$$
\begin{aligned}
& P_{M}(z \mid \ell, m, n, k, j, i) \\
& \quad=P\left[\mathbf{M}_{a}(\ell, k), \mathbf{M}_{d}(m, j), \mathbf{M}(n, i)\right] .
\end{aligned}
$$

Let $P_{\text {out }}(M \mid z)$ and $C_{\text {end }}(M \mid z)$ represent the conditional outage probability and end-to-end capacity. Given $\ell, m$, and $n$, it follows that

$$
\begin{aligned}
P_{\text {out }} & (M \mid \ell, m, n) \\
= & \sum_{i=1}^{\left(\begin{array}{c}
N \\
n
\end{array}\right)} \sum_{j=1}^{\left(\begin{array}{c}
n \\
m
\end{array}\right)} \sum_{k=1}^{\left(\begin{array}{c}
m \\
\ell
\end{array}\right)} P_{\text {out }}(M \mid z) P_{M}(z \mid \ell, m, n, k, j, i)
\end{aligned}
$$

and

$$
\begin{aligned}
C_{\text {end }} & (M \mid \ell, m, n) \\
= & \sum_{i=1}^{\left(\begin{array}{c}
N \\
n
\end{array}\right)} \sum_{j=1}^{\left(\begin{array}{c}
n \\
m
\end{array}\right)} \sum_{k=1}^{\left(\begin{array}{c}
m \\
\ell
\end{array}\right)} C_{\text {end }}(M \mid z) P_{M}(z \mid \ell, m, n, k, j, i),
\end{aligned}
$$

respectively. Finally, the overall outage probability and endto-end capacity for the case with at least one masquerader in $\mathscr{D}(S)$ can be expressed as

$$
P_{\text {out }}(M)=\sum_{n=1}^{N} \sum_{m=1}^{n} \sum_{\ell=1}^{m} P_{\text {out }}(M \mid \ell, m, n)
$$

and

$$
C_{\text {end }}(M)=\sum_{n=1}^{N} \sum_{m=1}^{n} \sum_{\ell=1}^{m} C_{\text {end }}(M \mid \ell, m, n) .
$$

$P_{\text {out }}(M \mid z)$ and $C_{\text {end }}(M \mid z)$ are derived as follows.

(1). Recall that there are $\ell$ active-and-decodable masqueraders in the subset $\mathbf{M}_{a}(\ell, k), \forall k=1, \ldots,\left(\begin{array}{c}m \\ \ell\end{array}\right)$. When $\ell>1$, one of the $\ell$ active-and-decodable masqueraders is randomly selected to forward packets during Phase II's transmission period. Thus, the conditional outage probability should be averaged over these $\ell$ cases. Then, $P_{\text {out }}(M \mid z)$ can be expressed as

$$
\begin{aligned}
P_{\text {out }} & (M \mid z)=\frac{1}{\ell} \sum_{z \in \mathbf{M}_{a}(\ell, k)} P\left[C_{z}^{I I}<C_{t h}\right] \\
= & \frac{1}{\ell} \sum_{z \in \mathbf{M}_{a}(\ell, k)} P\left[\frac{1}{2} \log _{2}\left(1+y \gamma_{S}+x \gamma_{R}\right)<C_{t h}\right] \\
= & \frac{1}{\ell} \sum_{z \in \mathbf{M}_{a}(\ell, k)} P\left[\left(x \gamma_{R}+y \gamma_{S}\right)<\lambda_{t h}^{\prime}\right] \\
= & \frac{1}{\ell} \sum_{z \in \mathbf{M}_{a}(\ell, k)} P\left[x<\frac{\lambda_{t h}^{\prime}-y \gamma_{S}}{\gamma_{R}}\right],
\end{aligned}
$$

where $\lambda_{t h}^{\prime}=2^{2 C_{t h}}-1$. Substituting (2) and (3) into (23) gives

$$
\begin{aligned}
& P_{\text {out }}(M \mid z)=\frac{1}{\ell} \sum_{z \in \mathbf{M}_{a}(\ell, k)} \int_{0}^{\lambda_{t h}^{\prime} / \gamma_{S}} F_{X}\left(\frac{\lambda_{t h}^{\prime}-y \gamma_{S}}{\gamma_{R}}\right) f_{Y}(y) d y \\
& P_{\text {out }}(M \mid z)=\frac{1}{\ell} \\
& \cdot \sum_{z \in \mathbf{M}_{a}(\ell, k)} \int_{0}^{\lambda_{t h}^{\prime} / \gamma_{S}} \int_{0}^{\left(\lambda_{t h}^{\prime}-y \gamma_{s}\right) / \gamma_{R}} \frac{\xi}{\sqrt{2 \pi} \widehat{\sigma} x} \exp \left(-\frac{\left(\xi \ln x-\widehat{\mu}_{x}\right)^{2}}{2 \widehat{\sigma}^{2}}\right) \\
& \cdot \frac{\xi}{\sqrt{2 \pi} \widehat{\sigma} y} \exp \left(-\frac{\left(\xi \ln y-\widehat{\mu}_{y}\right)^{2}}{2 \widehat{\sigma}^{2}}\right) d x d y
\end{aligned}
$$

Let $u=\left(\xi \ln x-\widehat{\mu}_{x}\right) / \widehat{\sigma}$ and $v=\left(\xi \ln y-\widehat{\mu}_{y}\right) / \widehat{\sigma}$. Then, (25) can be rewritten as

$$
\begin{aligned}
& P_{\text {out }}(M \mid z)=\frac{1}{\ell} \\
& \quad \sum_{z \in \mathbf{M}_{a}(\ell, k)} \int_{-\infty}^{\left(\xi \ln \left(\lambda_{t h}^{\prime} / \gamma_{S}\right)-\widehat{\mu}_{y}\right) / \widehat{\sigma}} \int_{-\infty}^{\left(\xi \ln y^{\prime}-\widehat{\mu}_{x}\right) / \widehat{\sigma}} \frac{1}{\sqrt{2 \pi}} \exp \left(-\frac{u^{2}}{2}\right) \frac{1}{\sqrt{2 \pi}} \\
& \quad \cdot \exp \left(-\frac{v^{2}}{2}\right) d u d v
\end{aligned}
$$

where

$$
y^{\prime}=\left(\frac{\lambda_{t h}^{\prime}-\gamma_{S} \exp \left(\left(v \widehat{\sigma}+\widehat{\mu}_{y}\right) / \xi\right)}{\gamma_{R}}\right) .
$$


Since there is no closed form expression for (26), it can only be reduced to

$$
\begin{aligned}
& P_{\text {out }}(M \mid z)=\frac{1}{\ell} \\
& \cdot \sum_{z \in \mathbf{M}_{a}(\ell, k)} \int_{-\infty}^{\left(\xi \ln \left(\lambda_{t h}^{\prime} / \gamma_{S}\right)-\widehat{\mu}_{y}\right) / \widehat{\sigma}} Q\left(\frac{\widehat{\mu}_{x}-\xi \ln y^{\prime}}{\widehat{\sigma}}\right) \frac{1}{\sqrt{2 \pi}} \\
& \cdot \exp \left(-\frac{v^{2}}{2}\right) d v .
\end{aligned}
$$

(2). Similar to (23), $C_{\text {end }}(M \mid z)$ can be expressed as

$$
C_{\text {end }}(M \mid z)=\frac{1}{\ell} \sum_{z \in \mathbf{M}_{a}(\ell, k)} E\left[\frac{1}{2} \log _{2}\left(1+x \gamma_{R}+y \gamma_{S}\right)\right]
$$

where $E[\cdot]$ is the operator to take expectation. Substituting (2) into (29) renders (30).

$$
\begin{aligned}
& C_{\text {end }}(M \mid z)=\frac{1}{\ell} \\
& \cdot \sum_{z \in \mathbf{M}_{a}(\ell, k)} \int_{0}^{\infty} \int_{0}^{\infty} \frac{1}{2} \log _{2}\left(1+x \gamma_{R}+y \gamma_{S}\right) \\
& \cdot \frac{\xi}{\sqrt{2 \pi} \widehat{\sigma} x} \exp \left(-\frac{\left(\xi \ln x-\widehat{\mu}_{x}\right)^{2}}{2 \widehat{\sigma}^{2}}\right) \\
& \quad \times \frac{\xi}{\sqrt{2 \pi} \widehat{\sigma} y} \exp \left(-\frac{\left(\xi \ln y-\widehat{\mu}_{y}\right)^{2}}{2 \widehat{\sigma}^{2}}\right) d x d y
\end{aligned}
$$

Let $u=\left(\xi \ln x-\widehat{\mu}_{x}\right) /(\sqrt{2} \widehat{\sigma})$ and $v=\left(\xi \ln y-\widehat{\mu}_{y}\right) /(\sqrt{2} \widehat{\sigma})$ which leads to

$$
C_{\text {end }}(M \mid z)=\frac{1}{\ell} \sum_{z \in \mathbf{M}_{a}(\ell, k)} \int_{-\infty}^{\infty} \int_{-\infty}^{\infty} \frac{1}{2} \log _{2}\left[1+\gamma_{R} \exp \left(\frac{\sqrt{2} \widehat{\sigma} u+\widehat{\mu}_{x}}{\xi}\right)+\gamma_{S} \exp \left(\frac{\sqrt{2} \widehat{\sigma} v+\widehat{\mu}_{y}}{\xi}\right)\right] \times \frac{1}{\pi} e^{-v^{2}} e^{-u^{2}} d u d v .
$$

It is known that the Hermite polynomial approach can be applied to calculate the following integration:

$$
\int_{-\infty}^{\infty} f(x) e^{-x^{2}} d x=\sum_{\eta=1}^{N_{H}} \omega_{\eta} f\left(t_{\eta}\right)
$$

where $t_{\eta}$ and $\omega_{\eta}$ are the abscissas and the weight factor of the Hermite polynomials with order $N_{H}$, respectively [20]. Applying (32) into (31) renders

$$
\begin{aligned}
& C_{\text {end }}(M \mid z) \cong \frac{1}{\ell} \sum_{z \in \mathbf{M}_{a}(\ell, k)} \sum_{\eta=1}^{N_{H}} \sum_{\kappa=1}^{N_{H}} \frac{\omega_{\eta} \omega_{\kappa}}{2} \log _{2}[1 \\
& \quad+\gamma_{R} \exp \left(\frac{\sqrt{2} \widehat{\sigma} t_{\eta}+\widehat{\mu}_{x}}{\xi}\right) \\
& \left.\quad+\gamma_{S} \exp \left(\frac{\sqrt{2} \widehat{\sigma} t_{\kappa}+\widehat{\mu}_{y}}{\xi}\right)\right]
\end{aligned}
$$

4.2. No Active Masqueraders in the Nonempty $\mathscr{D}(S)$. According to Section 3.2, three cases should be considered in this scenario; i.e., the $z$-th relay is selected from $\mathbf{O}_{d}(n, i)$, $\mathbf{O}_{d}(m, j)$, or $\mathbf{O}_{d}(\ell, k)$. Similar to Section 4.1 , let $P_{\text {out }}^{\mathscr{A}}(O)$, $P_{\text {out }}^{\mathscr{B}}(O)$, and $P_{\text {out }}^{\mathscr{C}}(O)$ denote the outage probability for these cases. Also, $C_{\text {end }}^{\mathscr{A}}, C_{\text {end }}^{\mathscr{B}}$, and $C_{\text {end }}^{\mathscr{C}}$ are the corresponding endto-end capacity, respectively. Then, we can have

$$
P_{\text {out }}(O)=P_{\text {out }}^{\mathscr{A}}(O)+P_{\text {out }}^{\mathscr{B}}(O)+P_{\text {out }}^{\mathscr{C}}(O)
$$

and

$$
C_{\text {end }}(O)=C_{\text {end }}^{\mathscr{A}}(O)+C_{\text {end }}^{\mathscr{B}}(O)+C_{\text {end }}^{\mathscr{C}}(O)
$$

(1) Case A: No Relays Become the Masqueraders. Denote $P_{\text {out }}^{\mathscr{A}}(O \mid z)$ and $C_{\text {end }}^{\mathscr{A}}(O \mid z)$ the outage probability and endto-end capacity when the $z$-th relay is selected from $\mathbf{O}_{d}(n, i)$ under the condition of $\mathbf{O}(N)$. Similar to the procedures of deriving (21) and (22), the average outage probability and end-to-end capacity can be expressed as

$$
\begin{aligned}
& P_{\text {out }}^{\mathscr{A}}(O) \\
& =\sum_{n=1}^{N} \sum_{i=1}^{\left(\begin{array}{l}
N \\
n
\end{array}\right)} P_{\text {out }}^{\mathscr{A}}(O \mid z) P\left[\mathbf{O}_{d}(n, i) \mid \mathbf{O}(N)\right] P[\mathbf{O}(N)]
\end{aligned}
$$

and

$$
\begin{aligned}
& C_{\text {end }}^{\mathscr{A}}(O)=\sum_{n=1}^{N} \sum_{i=1}^{\left(\begin{array}{l}
N \\
n
\end{array}\right)} C_{\text {end }}^{\mathscr{A}}(O \mid z) P\left[\mathbf{O}_{d}(n, i) \mid \mathbf{O}(N)\right] \\
& \cdot P[\mathbf{O}(N)],
\end{aligned}
$$

respectively.

(a) . Regardless of which relay is selected from $\mathbf{O}_{d}(n, i)$ in Phase II, an outage event can occur when the end-to-end capacity of all the relays belonging to $\mathbf{O}_{d}(n, i)$ is below the target value, i.e., $C_{z}^{I I}<C_{t h} \forall z \in \mathbf{O}_{d}(n, i)$. Let $\widehat{F}_{X}(\zeta)$ denote the $c d f$ of this case. Referring to (3), the outage probability with a given $y$ can be written as

$$
\begin{aligned}
\widehat{F}_{X}\left(\frac{\lambda_{t h}^{\prime}-y \gamma_{S}}{\gamma_{R}}\right) \\
\quad=\prod_{z \in \mathbf{O}_{d}(n, i)} Q\left(\frac{\widehat{\mu}_{x}-\xi \ln \left(\left(\lambda_{t h}^{\prime}-y \gamma_{S}\right) / \gamma_{R}\right)}{\widehat{\sigma}}\right) .
\end{aligned}
$$


Then, similar to (24), the outage probability in this case can be expressed as

$$
\begin{aligned}
& P_{\text {out }}^{\mathscr{A}}(O \mid z)=\int_{0}^{\lambda_{t h}^{\prime} / \gamma_{S}} \widehat{F}_{X}\left(\frac{\lambda_{t h}^{\prime}-y \gamma_{S}}{\gamma_{R}}\right) f_{Y}(y) d y \\
& =\int_{0}^{\lambda_{t h}^{\prime} / \gamma_{S}} \prod_{z \in \mathbf{O}_{d}(n, i)} Q\left(\frac{\widehat{\mu}_{x}-\xi \ln \left(\left(\lambda_{t h}^{\prime}-y \gamma_{S}\right) / \gamma_{R}\right)}{\widehat{\sigma}}\right)
\end{aligned}
$$

$\cdot f_{Y}(y) d y$

Applying the same procedures of deriving (28) gives

$$
\begin{aligned}
& P_{\text {out }}^{\mathscr{A}}(O \mid z) \\
& \quad=\int_{-\infty}^{\left(\xi \ln \left(\lambda_{t h}^{\prime} / \gamma_{s}\right)-\widehat{\mu}_{y}\right) / \widehat{\sigma}} \prod_{z \in \mathbf{O}_{d}(n, i)} Q\left(\frac{\widehat{\mu}_{x}-\xi \ln y^{\prime}}{\widehat{\sigma}}\right) \\
& \quad \cdot \frac{1}{\sqrt{2 \pi}} \exp \left(-\frac{v^{2}}{2}\right) d v,
\end{aligned}
$$

where $y^{\prime}$ is the same as that in (27).

(b). In this case, the end-to-end capacity can be written as

$$
\begin{aligned}
& C_{\text {end }}^{\mathscr{A}}(O \mid z)=\int_{0}^{\infty} \int_{0}^{\infty} \frac{1}{2} \log _{2}\left(1+x \gamma_{R}+y \gamma_{S}\right) \\
& \quad \times f_{Y}(y) \widehat{f}_{X}(x) d x d y,
\end{aligned}
$$

where $\widehat{f}_{X}(\zeta)$ represents the corresponding $p d f$ of (38). Taking derivation of (38) gives

$$
\begin{aligned}
& \widehat{f}_{X}(\zeta)=\sum_{z \in \mathbf{O}_{d}(n, i)} \frac{\xi}{\sqrt{2 \pi} \widehat{\sigma} \zeta} \exp \left(-\frac{\left(\xi \ln \zeta-\widehat{\mu}_{x}\right)^{2}}{2 \widehat{\sigma}^{2}}\right) \\
& \times \prod_{z^{\prime} \in \mathbf{O}_{d}(n, i), z^{\prime} \neq z} Q\left(\frac{\widehat{\mu}_{x^{\prime}}-\xi \ln \zeta}{\widehat{\sigma}}\right),
\end{aligned}
$$

where $\mu_{x^{\prime}}$ associated with the $z^{\prime}$-th relay in $\mathbf{O}_{d}(n, i)$ is similar to $\mu_{x}$ associated with the $z$-th relay in $\mathbf{O}_{d}(n, i)$. Then, the average end-to-end capacity can be expressed as

$$
\begin{aligned}
& C_{\text {end }}^{\mathscr{A}}(O \mid z)=\sum_{z \in \mathbf{O}_{d}(n, i)} \int_{0}^{\infty} \int_{0}^{\infty} \frac{1}{2} \log _{2}\left(1+x \gamma_{R}+y \gamma_{S}\right) \\
& \cdot \frac{\xi}{\sqrt{2 \pi} \hat{\sigma} x} \exp \left(-\frac{\left(\xi \ln x-\widehat{\mu}_{x}\right)^{2}}{2 \widehat{\sigma}^{2}}\right) \\
& \cdot \prod_{z^{\prime} \in \mathbf{O}_{d}(n, i), z^{\prime} \neq z} Q\left(\frac{\widehat{\mu}_{x^{\prime}}-\xi \ln x}{\widehat{\sigma}}\right) \\
& \times \frac{\xi}{\sqrt{2 \pi} \hat{\sigma} y} \exp \left(-\frac{\left(\xi \ln y-\widehat{\mu}_{y}\right)^{2}}{2 \widehat{\sigma}^{2}}\right) d x d y
\end{aligned}
$$

Letting $u=\left(\xi \ln x-\widehat{\mu}_{x}\right) /(\sqrt{2} \widehat{\sigma})$ and $v=\left(\xi \ln y-\widehat{\mu}_{y}\right) /(\sqrt{2} \widehat{\sigma})$ gives

Applying the Hermite polynomial approach into (44) renders

$$
\begin{aligned}
& C_{\text {end }}^{\mathscr{A}}(O \mid z) \cong \sum_{\eta=1}^{N_{H}} \sum_{\kappa=1}^{N_{H}} \sum_{z \in \mathbf{O}_{d}(n, i)} \frac{\omega_{\eta} \omega_{\kappa}}{2} \log _{2}\left[1+\gamma_{R} \exp \left(\frac{\sqrt{2} \widehat{\sigma} t_{\eta}+\widehat{\mu}_{x}}{\xi}\right)+\gamma_{S} \exp \left(\frac{\sqrt{2} \widehat{\sigma} t_{\kappa}+\widehat{\mu}_{y}}{\xi}\right)\right] \\
& \times \prod_{z^{\prime} \in \mathbf{O}_{d}(n, i), z^{\prime} \neq z} Q\left(\frac{\widehat{\mu}_{x^{\prime}}-\widehat{\mu}_{x}-t_{\eta} \sqrt{2} \widehat{\sigma}}{\widehat{\sigma}}\right) .
\end{aligned}
$$

(2) Case $\mathscr{B}:$ No Masqueraders Are Decodable. Denote by $P_{\text {out }}^{\mathscr{B}}(O \mid z)$ and $C_{\text {end }}^{\mathscr{B}}(O \mid z)$ the conditional outage probability and end-to-end capacity when the $z$-th relay is selected from $\mathbf{O}_{d}(m, j)$ under the conditions of $\overline{\mathbf{M}}_{d}(n, i)$ and $\mathbf{M}(n, i)$. Then, the outage probability and end-to-end capacity can be expressed as (46) and (47), respectively. 


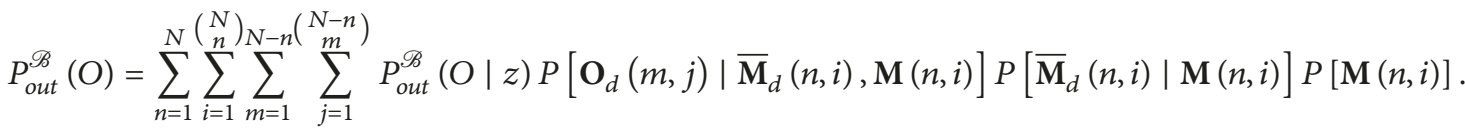

$$
\begin{aligned}
& C_{\text {end }}^{\mathscr{B}}(O)=\sum_{n=1}^{N} \sum_{i=1}^{\left(\begin{array}{c}
N \\
n
\end{array}\right.} \sum_{m=1}^{N-n} \sum_{j=1}^{\left(\begin{array}{c}
N-n \\
m
\end{array}\right)} C_{\text {end }}^{\mathscr{B}}(O \mid z) P\left[\mathbf{O}_{d}(m, j) \mid \overline{\mathbf{M}}_{d}(n, i), \mathbf{M}(n, i)\right] P\left[\overline{\mathbf{M}}_{d}(n, i) \mid \mathbf{M}(n, i)\right] P[\mathbf{M}(n, i)] .
\end{aligned}
$$

Applying the same procedures as mentioned in Case $\mathscr{A}$ by replacing $\mathbf{O}_{d}(n, i)$ in (40) and (45) with $\mathbf{O}_{d}(m, j)$ gives the conditional outage probability $P_{\text {out }}^{\mathscr{B}}(O \mid z)$ and end-to-end capacity $C_{\text {end }}^{\mathscr{B}}(O \mid z)$ for this case.

(3) Case $\mathscr{C}$ : No Decodable Masqueraders Are Active. Similar to Cases $\mathscr{A}$ and $\mathscr{B}$, let $P_{\text {out }}^{\mathscr{C}}(O \mid z)$ and $C_{\text {end }}^{\mathscr{C}}(O \mid z)$ represent the conditional outage probability and endto-end capacity when the $z$-th relay is selected from $\mathbf{O}_{d}(\ell, k)$ under the conditions of $\overline{\mathbf{M}}_{a}(m, j), \mathbf{M}_{d}(m, j)$, and $\mathbf{M}(n, i)$. Moreover, the outage probability and end-to-end capacity can be expressed as (48) and (49), respectively.

$$
\begin{aligned}
P_{\text {out }}^{\mathscr{C}}(O)= & \sum_{n=1}^{N} \sum_{m=1}^{n} \sum_{\ell=1}^{N-n} \sum_{i=1}^{\left(\begin{array}{c}
N \\
n
\end{array}\right)} \sum_{j=1}^{\left(\begin{array}{c}
n \\
m
\end{array}\right)} \sum_{k=1}^{\left(\begin{array}{c}
N-n \\
\ell
\end{array}\right)} P_{\text {out }}^{\mathscr{C}}(O \mid z) P\left[\mathbf{O}_{d}(\ell, k) \mid \overline{\mathbf{M}}_{a}(m, j), \mathbf{M}_{d}(m, j), \mathbf{M}(n, i)\right] \\
& \times P\left[\overline{\mathbf{M}}_{a}(m, j) \mid \mathbf{M}_{d}(m, j), \mathbf{M}(n, i)\right] P\left[\mathbf{M}_{d}(m, j) \mid \mathbf{M}(n, i)\right] P[\mathbf{M}(n, i)] . \\
C_{\text {end }}^{\mathscr{C}}(O)= & \sum_{n=1}^{N} \sum_{m=1}^{n} \sum_{\ell=1}^{N-n} \sum_{i=1}^{n} \sum_{j=1}^{N} \sum_{k=1}^{\left(\begin{array}{c}
n \\
m
\end{array}\right)} C_{\text {end }}^{\mathscr{C}}(O \mid z) P\left[\mathbf{O}_{d}(\ell, k) \mid \overline{\mathbf{M}}_{a}(m, j), \mathbf{M}_{d}(m, j), \mathbf{M}(n, i)\right] \\
& \times P\left[\overline{\mathbf{M}}_{a}(m, j) \mid \mathbf{M}_{d}(m, j), \mathbf{M}(n, i)\right] P\left[\mathbf{M}_{d}(m, j) \mid \mathbf{M}(n, i)\right] P[\mathbf{M}(n, i)] .
\end{aligned}
$$

At last, replacing $\mathbf{O}_{d}(n, i)$ in (40) and (45) with $\mathbf{O}_{d}(\ell, k)$ gives $P_{\text {out }}^{\mathscr{C}}(O \mid z)$ and $C_{\text {end }}^{\mathscr{C}}(O \mid z)$.

4.3. Empty Decodable Set $\mathscr{D}(S)$. Denote by $\mathscr{D}(S)=\emptyset$ the empty decodable set. Then, we can have the occurring probability for this case as

$$
P[\mathscr{D}(S)=\emptyset]=\prod_{\eta \in \mathbf{N}} Q\left(\frac{\widehat{\mu}_{\eta}^{I I}-\xi \ln \lambda_{t h}}{\widehat{\sigma}}\right) .
$$

(1). With $\mathscr{D}(S)=\emptyset$, the outage probability can be expressed as

$$
P_{\text {out }}(\emptyset)=P\left[C_{S}<C_{\text {th }} \mid \mathscr{D}(S)=\emptyset\right] P[\mathscr{D}(S)=\emptyset],
$$

where $P\left[C_{S}<C_{t h} \mid \mathscr{D}(S)=\emptyset\right]$ can also be derived as

$$
\begin{aligned}
P & {\left[C_{S}<C_{t h} \mid \mathscr{D}(S)=\emptyset\right] } \\
& =P\left[\log _{2}\left(1+y \gamma_{S}\right)<C_{t h} \mid \mathscr{D}(S)=\emptyset\right] \\
& =P\left[y<\lambda_{t h} \mid \mathscr{D}(S)=\emptyset\right]=Q\left(\frac{\widehat{\mu}_{y}-\xi \ln \lambda_{t h}}{\widehat{\sigma}}\right),
\end{aligned}
$$

where $\lambda_{t h}=\left(2^{C_{t h}}-1\right) / \gamma_{S}$.
(2). The capacity can be expressed as

$$
\begin{aligned}
& C_{\text {end }}(\emptyset) \\
& \quad=E\left[\log _{2}\left(1+y \gamma_{S}\right) \mid \mathscr{D}(S)=\emptyset\right] P[\mathscr{D}(S)=\emptyset],
\end{aligned}
$$

where $E\left[\log _{2}\left(1+y \gamma_{S}\right) \mid \mathscr{D}(S)=\emptyset\right]$ can be expressed as

$$
\begin{gathered}
E\left[\log _{2}\left(1+y \gamma_{S}\right) \mid \mathscr{D}(S)=\emptyset\right]=\int_{0}^{\infty} \log _{2}\left(1+y \gamma_{S}\right) \\
\cdot \frac{\xi}{\sqrt{2 \pi} \hat{\sigma} y} \exp \left(-\frac{\left(\xi \ln y-\widehat{\mu}_{S}\right)^{2}}{2 \widehat{\sigma}^{2}}\right) d x
\end{gathered}
$$

Following the procedure of deriving (33), we can have the numerical expression of (54) as

$$
\begin{aligned}
E & {\left[\log _{2}\left(1+y \gamma_{S}\right) \mid \mathscr{D}(S)=\emptyset\right] } \\
& \cong \sum_{\eta=1}^{N_{H}} \omega_{\eta} \log _{2}\left[1+\gamma_{S} \exp \left(\frac{\sqrt{2} \widehat{\sigma} t_{\eta}+\widehat{\mu}_{y}}{\xi}\right)\right] .
\end{aligned}
$$

\section{Numerical Results}

In this section, the relay-assisted D2D and cellular networks are considered to evaluate the exactness of the analytical model and investigate the performance degradation caused 


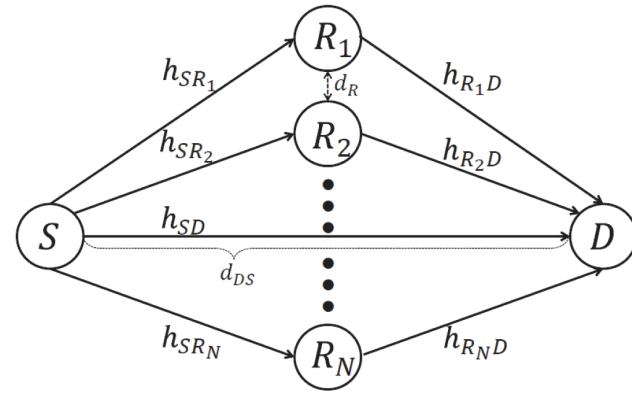

(a) Vertically deployed relays

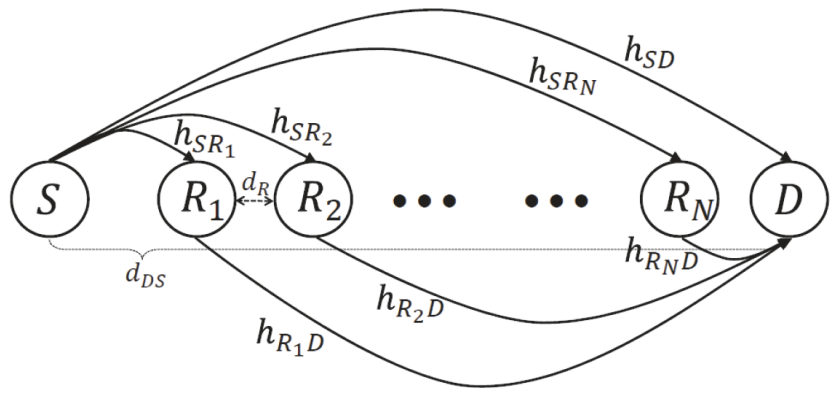

(b) Horizontally deployed relays

FiguRE 2: Relay-assisted D2D network with $N$ (a) vertically and (b) horizontally deployed relays.

by the masquerading attack. In either scenario, the unitvariance Rayleigh fading is assumed. Also, the path loss exponent is 3.5 and the standard deviation of the Log-Normal shadowing is 6 in the $\mathrm{dB}$ domain. All the simulation results are obtained by averaging over 200,000 rounds.

5.1. D2D Network. Here, the vertical deployment of the relay network (as shown in Figure 2(a)) is firstly applied for investigating the impact of the masquerading attack. Then, the horizontal deployment of Figure 2(b) is used to quantitatively investigate the effect of the location of a single designated masquerader. As shown in the figures, the distance between $S$ and $D$ is $d_{D S}=1000 \mathrm{~m}$; and that between two neighboring relays is $d_{R}=50 \mathrm{~m}$. The transmission power of the source $P_{S}$ is set so that the thermal noise outage $\left(O_{N}\right)$ at the destination can be 0.2 [19]. Similarly, the transmission power of each relay $P_{R}$ is set so that $O_{N}=0.2$ can be achieved by the central relay (e.g., $R_{2}$ with $N=3$ or $R_{3}$ with $N=5$ ). Note that the required SNR corresponding to $O_{N}=0.2$ is defined as $0 \mathrm{~dB}$, whereas, for the purpose of evaluating the outage probability, the SNR threshold is $8 \mathrm{~dB}$. Also, the capacity threshold $C_{t h}$ in Sections 2, 3, and 4 can be obtained by substituting the SNR threshold into the wellknown equation of Shannon capacity.

Figure 6 shows the (a) outage probability and (b) average end-to-end capacity with respect to $P_{\beta}$ for the relay-assisted D2D network under the CELN channel environment, where $P_{\alpha}=0.5 ; N=3, N=5$, and $N=9$ relays are placed according to the vertical deployment as illustrated in Figure 2(a). Apparently, the analytic and simulation results match with each other quite well. Most importantly, as demonstrated in the figures, the masquerading attack can cause significant performance degradation. For example, as the $P_{\beta}$ increases from 0 to 0.4 , the outage probability for the case with $N=5$ can increase from 0.03 to 0.16 . In addition, it can lead to $16 \%$ capacity loss (from 2.95 to 2.49 bps/Hz). Moreover, as $P_{\beta}$ keeps growing, its impact becomes marginal. It should be noticed that the equivalent activity of the masquerading behavior for each relay is $P_{\alpha} \times P_{\beta}$, whereas the overall masquerading behavior across the whole network is $N \times P_{\alpha} \times P_{\beta}$. Thus, with $N=5, P_{\alpha}=0.5$, and $P_{\beta}=0.2$, solely $P_{\alpha} \times P_{\beta}=0.5 \times 0.2=10 \%$ (or overall $5 \times 0.5 \times 0.2=$
$50 \%$ ) equivalent activity of the masqueraders can cause $9.2 \%$ capacity loss (from 2.95 to 2.68 ), while the outage probability can increase from 0.03 to 0.1 . When the equivalent activity becomes $P_{\alpha} \times P_{\beta}=0.5 \times 1=50 \%$ (or overall $N \times P_{\alpha} \times P_{\beta}=5 \times$ $0.5 \times 1=250 \%)$, it ends in $25 \%$ capacity loss and unacceptably high outage probability of 0.23 (667\% rise).

This phenomenon can become deteriorated when the number of relays increases. For example, comparing the curve of $N=5$ at $P_{\beta}=0.4$, using $N=9$ relays can result in additional $6 \%$ capacity loss (from 2.49 to $2.34 \mathrm{bps} / \mathrm{Hz}$ ), and higher outage probability (increasing from 0.16 to 0.21 ). Moreover, as $P_{\beta}$ grows to one, $34 \%$ capacity loss can be resulted; and the outage probability can be extremely risen by $6310 \%$. It should be noticed that, in general, using more relays can contribute to a better system performance. However, the masqueraders seriously dilute the diversity gain. Thus, how to tackle the issue of masqueraders could be an important issue for the future generation of hyperdense relay networks. Figure 3 shows the (a) outage probability and (b) average end-to-end capacity with respect to $P_{\beta}$ for the relay-assisted D2D network under the CELN channel environment, where $P_{\alpha}=0.5 ; N=3, N=5$, and $N=9$ relays are placed according to the vertical deployment as illustrated in Figure 2(a).

Figure 4 shows the (a) outage probability and (b) average end-to-end capacity for the relay-assisted D2D network under the CELN environment with a single designated masquerader according to the horizontal deployment as illustrated in Figure 2(b), where $N=7$ and $P_{\beta}=1$. Note that the cases without masquerader mean that all the $N=7$ relays are ordinary ones, whereas the cases with masquerader mean that there is only one masquerader indicated by the horizontal axis. In addition to the similar phenomenon observed from Figure 6 (i.e., the significant performance degradation caused by the masquerader), one can also find that the masquerader located farther from the destination can cause severer performance degradation. This explains the lowest capacity and highest outage probability for the case of the first relay (i.e., $R_{1}$ ) being the designated masquerader. Therefore, it can be expected that a masquerader can possibly incur a serious bottleneck effect on the multihop transmissions in the future generation of wireless communication systems. 


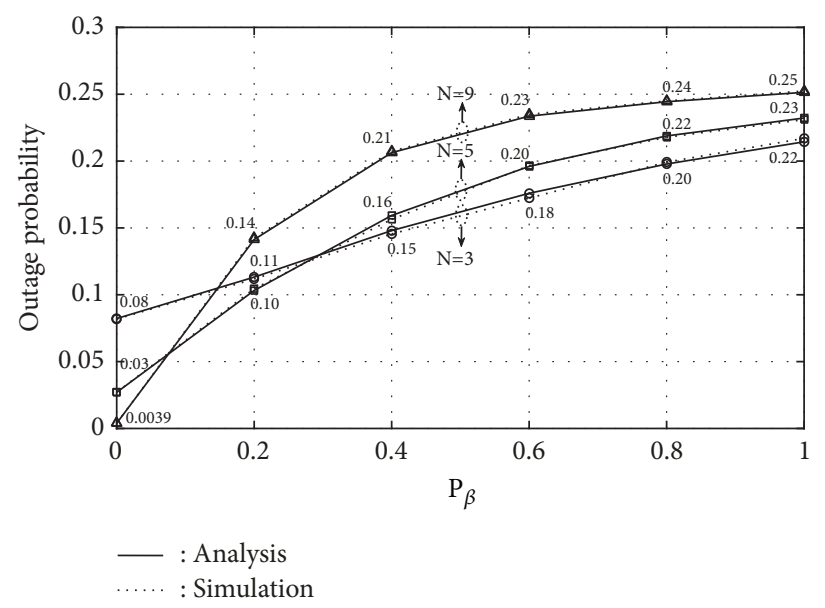

(a) Outage probability

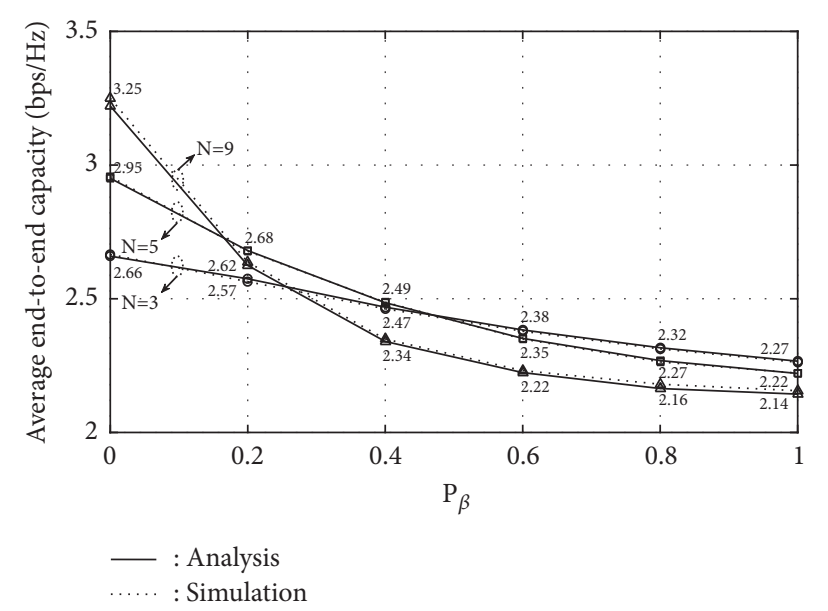

(b) Average end-to-end capacity

FIGURE 3: (a) Outage probability and (b) average end-to-end capacity with respect to $P_{\beta}$ for the relay-assisted D2D network under the CELN channel environment, where $P_{\alpha}=0.5 ; N=3, N=5$, and $N=9$ relays are placed according to the vertical deployment as illustrated in Figure 2(a).

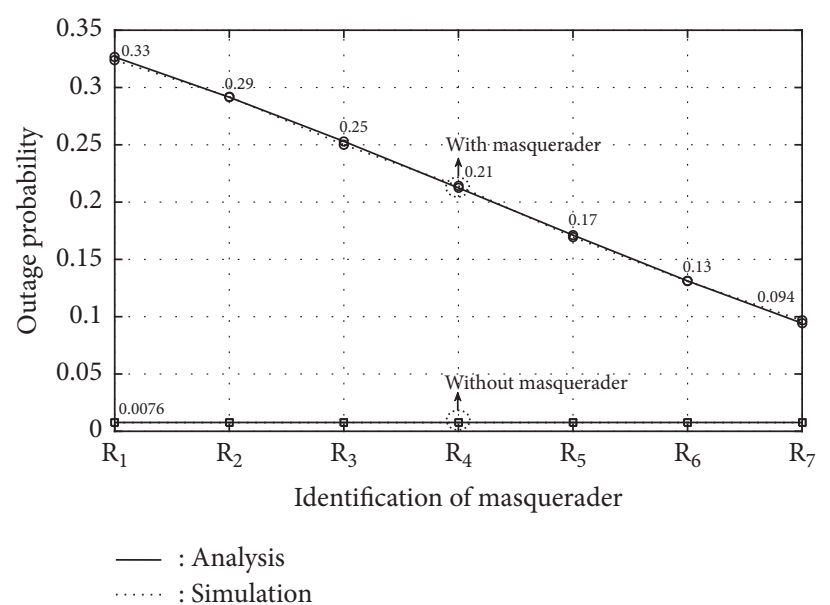

(a) Outage probability

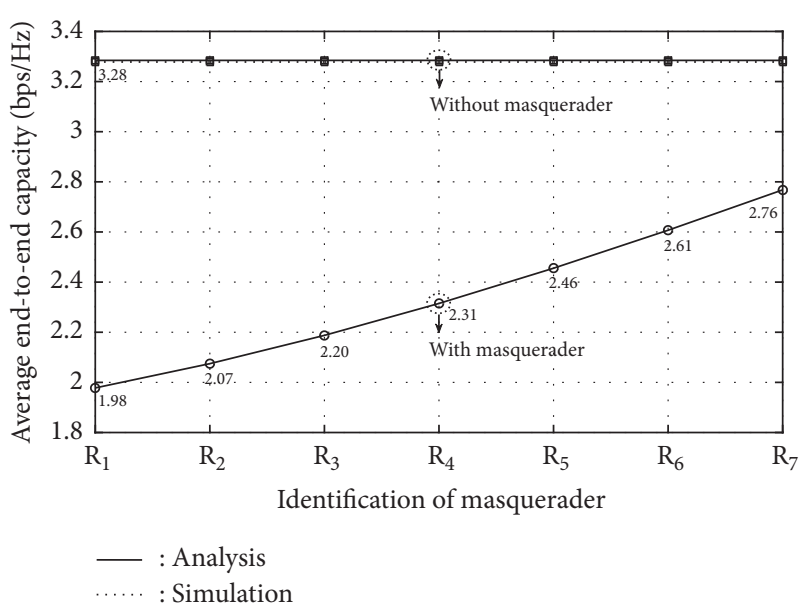

(b) Average end-to-end capacity

FIGURE 4: (a) Outage probability and (b) average end-to-end capacity for the relay-assisted D2D network under the CELN environment with a single designated masquerader according to the horizontal deployment as illustrated in Figure 2(b), where $N=7$ and $P_{\beta}=1$.

5.2. Cellular Network. In the cellular scenario, one cell with three $120^{\circ}$ sectors is considered. As per the parameter setting in Figure 1, the simulation is conducted for the network topologies illustrated in Figures 5(a) and 5(b). Therein, $N=3$ and 9 relays are fixed at the middles or uniformly distributed over its coverage area, respectively. In the latter case, the minimum distance between a relay and BS is $100 \mathrm{~m}$. Also, in both cases, the location of MS is uniformly spread over the sector. Since the analytical results are obtained by averaging over 100 randomly generated network topologies, the time required to calculate all the cases discussed in Sections 3 and 4 can be prohibitively prolonged when $N \geq 7$. Thus, only $N=3$ is considered for generating the analytical results. Firstly, the exactness of the analytical results can still be verified. Secondly, as observed in the D2D network, more stringent performance degradation can be incurred by the larger amount of relays. Thirdly, the masquerading attack can cause more significant performance degradation for the cases with uniformly distributed relays. For the example with $N=9$ uniformly distributed relays, serious masquerading attack can cause $30 \%$ capacity loss (from 4.93 to $3.46 \mathrm{bps} / \mathrm{Hz}$ ). However, the loss becomes $18 \%$ (from 3.71 to $3.05 \mathrm{bps} / \mathrm{Hz}$ ) when the relays are fixed at the middles of the sector. Note that the larger diversity gain can be obtained when the relays are "not fixed" at the middles of the sector (which explains the better performance for the cases with uniformly distributed relays as well). However, the masquerading attack dilutes the achievable diversity gain and causes larger performance degradation for the cases with larger diversity gain (as aforementioned). 


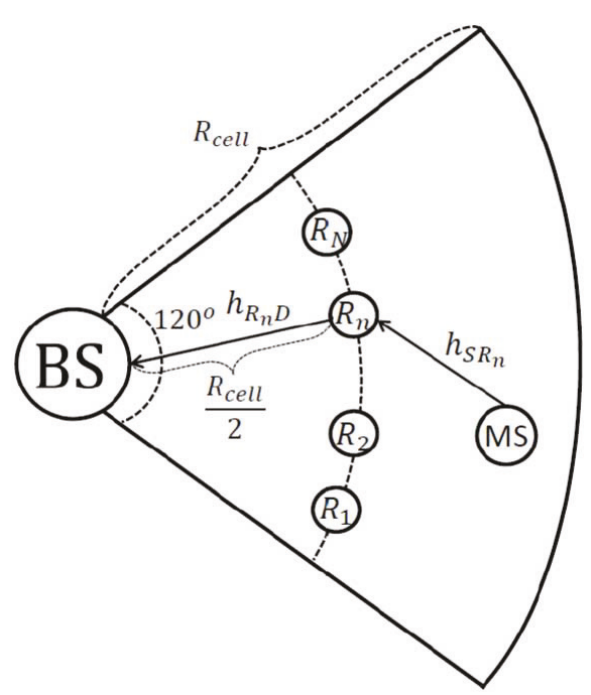

(a) A sector with fixed relays

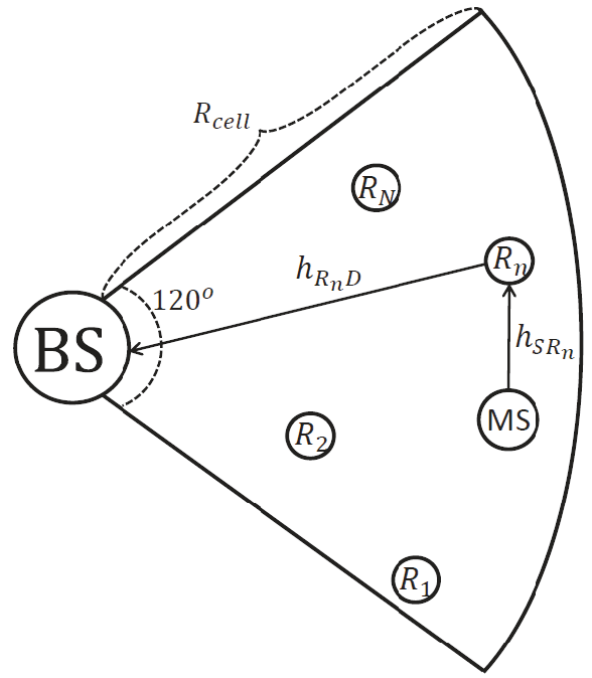

(b) A sector with uniformly distributed relays

FIGURE 5: A cellular sector with $N$ (a) fixed relays and (b) uniformly distributed relays.

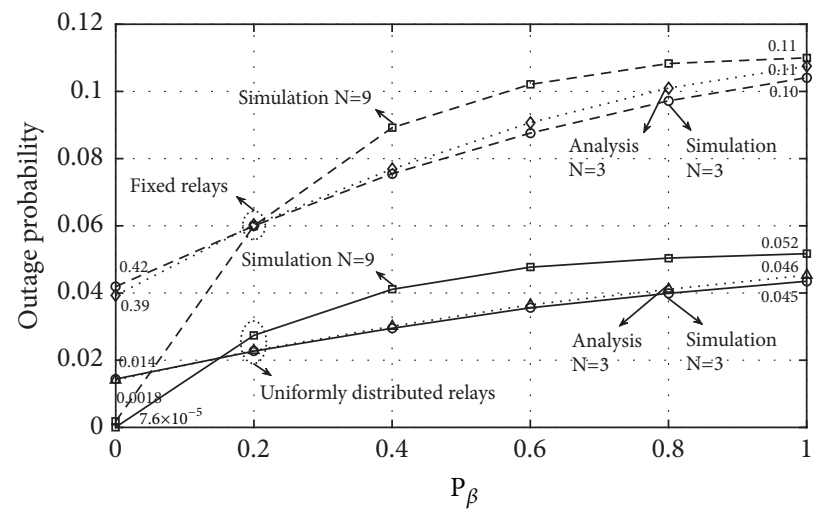

(a) Outage probability

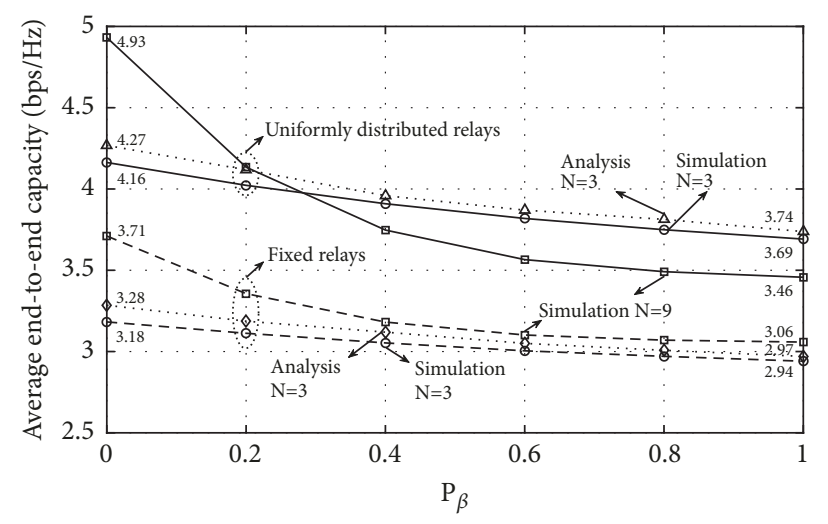

(b) Average end-to-end capacity

FIGURE 6: (a) Outage probability and (b) average end-to-end capacity with respect to $P_{\beta}$ for the cellular networks with fixed and uniformly distributed relays under the CELN channel environment, where $P_{\alpha}=0.5$; the numbers of relays are $N=3$ and $N=9$.

\section{Conclusions}

In this paper, we have defined the masquerading attack for the relay-assisted networks. For the purpose of numerically characterizing the masquerading attack, the mathematical expressions for the end-to-end capacity and outage probability have been derived. To make the discussions more complete, the CELN channel model was taken into account such that the geographic effects of the network topology can be captured. Moreover, the random masquerading behavior was considered as well, including the probability of a relay to become a masquerader and probability of a masquerader to become active. Via the analytical and simulation results, it was found that the masquerading attack can cause $34 \%$ and $30 \%$ capacity loss for considered D2D and cellular networks. Also, the corresponding outage probability can be extremely risen.
Nowadays, the necessity of relay-assisted transmission scheme for the next generation of wireless communication networks has been widely recognized. With the aid of relay, the performance of secondary users in the cognitive network can be improved [21]; the physical layer security in the largescale fifth-generation network can be strengthened [22]; the energy efficiency and link reliability for the vehicular ad hoc networks can be enhanced [23]; the cellular coverage area can be extended via multihop D2D communications $[6,7,24]$, especially for the millimeter-wave-based systems [25-27] and Internet-of-Things [28]; the high quality transmissions for the sensor network can be achieved [29] as well. However, based on the finding of this paper, the achievable diversity gain via relay transmissions will be seriously diluted under the masquerading attack. De facto, in addition to the DNPS protocol, any arbitrary relay networks (especially for the ones operating in the distributed mode) can encounter this 
kind of threat, which hypocritically forwards packets and removes the diversity gain in silence. Thus, how to evaluate and alleviate the impact of the masqueraders could be a critical issue to fully utilize the advantages of relay-assisted transmissions. This paper can be recognized as a first step to inspire the investigation of the masquerading attack for the relay networks.

\section{Data Availability}

All the analytical and numerical results can be reproduced by using the source codes at http://140.116.92.1/SourceCode/ WCMC/experiment. The Matlab codes used to support the findings of this study are available from the corresponding author upon request.

\section{Conflicts of Interest}

The author declares that there are no conflicts of interest regarding the publication of this paper.

\section{Acknowledgments}

This work was supported by the Ministry of Science and Technology, Taiwan, under Contract 105-2221-E-006-034.

\section{References}

[1] J. Xu, J. Wang, Y. Zhu et al., "Cooperative distributed optimization for the hyper-dense small cell deployment," IEEE Communications Magazine, vol. 52, no. 5, pp. 61-67, 2014.

[2] H. Shokri-Ghadikolaei, C. Fischione, P. Popovski, and M. Zorzi, "Design aspects of short-range millimeter-wave networks: A MAC layer perspective," IEEE Network, vol. 30, no. 3, pp. 8896, 2016.

[3] T. E. Bogale and L. B. Le, "Massive MIMO and mmWave for 5G wireless HetNet: potential benefits and challenges," IEEE Vehicular Technology Magazine, vol. 11, no. 1, pp. 64-75, 2016.

[4] M. Peng, Y. Liu, D. Wei, W. Wang, and H. Chen, "Hierarchical cooperative relay based heterogeneous networks," IEEE Wireless Communications Magazine, vol. 18, no. 3, pp. 48-56, 2011.

[5] M. Cardone, D. Tuninetti, and R. Knopp, "On the optimality of simple schedules for networks with multiple half-duplex relays," Institute of Electrical and Electronics Engineers Transactions on Information Theory, vol. 62, no. 7, pp. 4120-4134, 2016.

[6] L. Wei, R. Hu, Y. Qian, and G. Wu, "Key elements to enable millimeter wave communications for 5G wireless systems," IEEE Wireless Communications Magazine, vol. 21, no. 6, pp. 136143, 2014.

[7] J. Qiao, X. S. Shen, J. W. Mark, Q. Shen, Y. He, and L. Lei, "Enabling device-to-device communications in millimeterwave $5 \mathrm{G}$ cellular networks," IEEE Communications Magazine, vol. 53, no. 1, pp. 209-215, 2015.

[8] L. J. Rodriguez, N. H. Tran, T. Q. Duong, T. Le-Ngoc, M. Elkashlan, and S. Shetty, "Physical layer security in wireless cooperative relay networks: State of the art and beyond," IEEE Communications Magazine, vol. 53, no. 12, pp. 32-39, 2015.

[9] X. Wang, P. Hao, and L. Hanzo, "Physical-layer authentication for wireless security enhancement: Current challenges and future developments," IEEE Communications Magazine, vol. 54, no. 6, pp. 152-158, 2016.

[10] Y. Mao and M. Wu, "Tracing malicious relays in cooperative wireless communications," IEEE Transactions on Information Forensics and Security, vol. 2, no. 2, pp. 198-212, 2007.

[11] S. Dehnie and N. Memon, "Detection of misbehavior in cooperative diversity," in Proceedings of theMilitary Communications Conference (MILCOM), pp. 1-5, 2008.

[12] H. Zhu, X. Lin, R. Lu, Y. Fan, and X. Shen, "Smart: a secure multilayer credit-based incentive scheme for delay-tolerant networks," IEEE Transactions on Vehicular Technology, vol. 58, no. 8, pp. 4628-4639, 2009.

[13] L.-C Lo and W.-J Huang, "Misbehavior detection without channel information in cooperative networks," in Proceedings of the 2011 IEEE Vehicular Technology Conference (VTC Fall), pp. 1-5, San Francisco, CA, USA, September 2011.

[14] Y.-M. Yi, L.-C. Lo, and W.-J. Huang, "On blind sequential detection of misbehaving relay," in Proceedings of the Signal and Information Processing Association Annual Summit and Conference (APSIPA ASC), pp. 1-4, 2012.

[15] A. Bletsas, A. Khisti, D. P. Reed, and A. Lippman, "A simple cooperative diversity method based on network path selection," IEEE Journal on Selected Areas in Communications, vol. 24, no. 3, pp. 659-672, 2006.

[16] R. Gangula and R. Bhattacharjee, "Performance of selection cooperation in the presence of a malicious relay," in Proceedings of the 2010 7th International Symposium on Wireless Communication Systems, ISWCS'10, pp. 408-412, September 2010.

[17] J. Zhang and J. G. Andrews, "Distributed antenna systems with randomness," IEEE Transactions on Wireless Communications, vol. 7, no. 9, pp. 3636-3646, 2008.

[18] W. Chang, "On the performance of the DNPS-based relay networks under attack by masquerader," in Proceedings of the 2017 IEEE Wireless Communications and Networking Conference, (WCNC 2017), pp. 1-6, March 2017.

[19] G. L. Stuber, Principles of Mobile Communications, Kluwer Academic Publishers, 2nd edition, 2001.

[20] M. Abramowitz and I. A. Stegun, Handbook of Mathematical Functions with Formulas, Graphs, and Mathematical Tables, Dover Publications, New York, NY, USA, 9th edition, 1970.

[21] J. Van Hecke, P. Del Fiorentino, V. Lottici, F. Giannetti, L. Vandendorpe, and M. Moeneclaey, "Distributed Dynamic Resource Allocation for Cooperative Cognitive Radio Networks with Multi-Antenna Relay Selection," IEEE Transactions on Wireless Communications, vol. 16, no. 2, pp. 1236-1249, 2017.

[22] C. Zhang, J. Ge, J. Li, F. Gong, and H. Ding, "Complexity-aware relay selection for $5 \mathrm{~g}$ large-scale secure two-way relay systems," IEEE Transactions on Vehicular Technology, vol. 66, no. 6, pp. 5461-5465, 2017.

[23] D. Tian, J. Zhou, Z. Sheng, M. Chen, Q. Ni, and V. C. Leung, "Self-Organized Relay Selection for Cooperative Transmission in Vehicular Ad-Hoc Networks," IEEE Transactions on Vehicular Technology, vol. 66, no. 10, pp. 9534-9549, 2017.

[24] J. Gui and J. Deng, "Multi-hop relay-aided underlay d2d communications for improving cellular coverage quality," IEEE Access, vol. 6, pp. 14318-14338, 2018.

[25] Y. Niu, L. Su, C. Gao, Y. Li, D. Jin, and Z. Han, "Exploiting Device-to-Device Communications to Enhance Spatial Reuse for Popular Content Downloading in Directional mmWave Small Cells," IEEE Transactions on Vehicular Technology, vol. 65, no. 7, pp. 5538-5550, 2016. 
[26] W. Chang and J.-C. Teng, "Energy efficient relay matching with bottleneck effect elimination power adjusting for full-duplex relay assisted D2D networks using mmWave technology," IEEE Access, vol. 6, 2018.

[27] K. Belbase, Z. Zhang, H. Jiang, and C. Tellambura, "Coverage analysis of millimeter wave decode-and-forward networks with best relay selection,” IEEE Access, vol. 6, pp. 22670-22683, 2018.

[28] G. Chen, J. Tang, and J. P. Coon, "Optimal routing for multihop social-based $\mathrm{d} 2 \mathrm{~d}$ communications in the internet of things," IEEE Internet of Things Journal, vol. 5, no. 3, pp. 1880-1889, 2018.

[29] X. Li, J. Liu, L. Yan, S. Han, and X. Guan, "Relay selection for underwater acoustic sensor networks: a multi-user multiarmed bandit formulation," IEEE Access, vol. 6, pp. 7839-7853, 2018. 


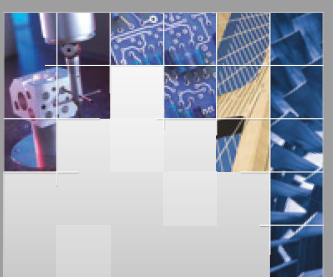

\section{Enfincering}
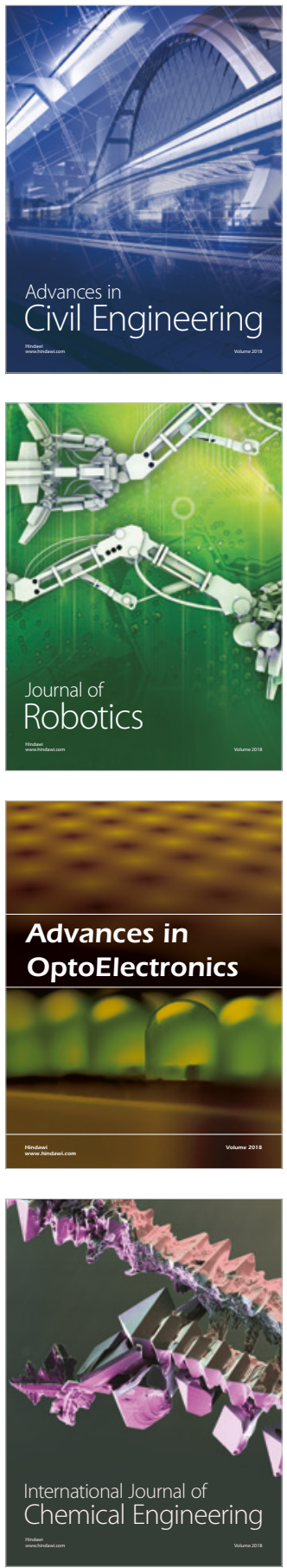

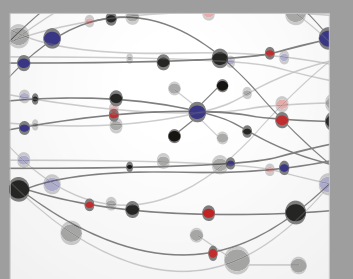

\section{Rotating \\ Machinery}

The Scientific World Journal

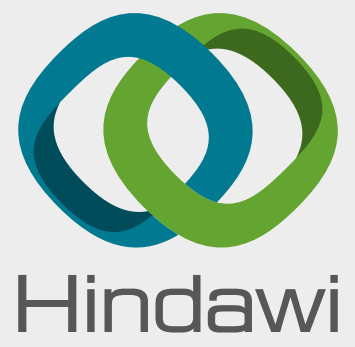

Submit your manuscripts at

www.hindawi.com
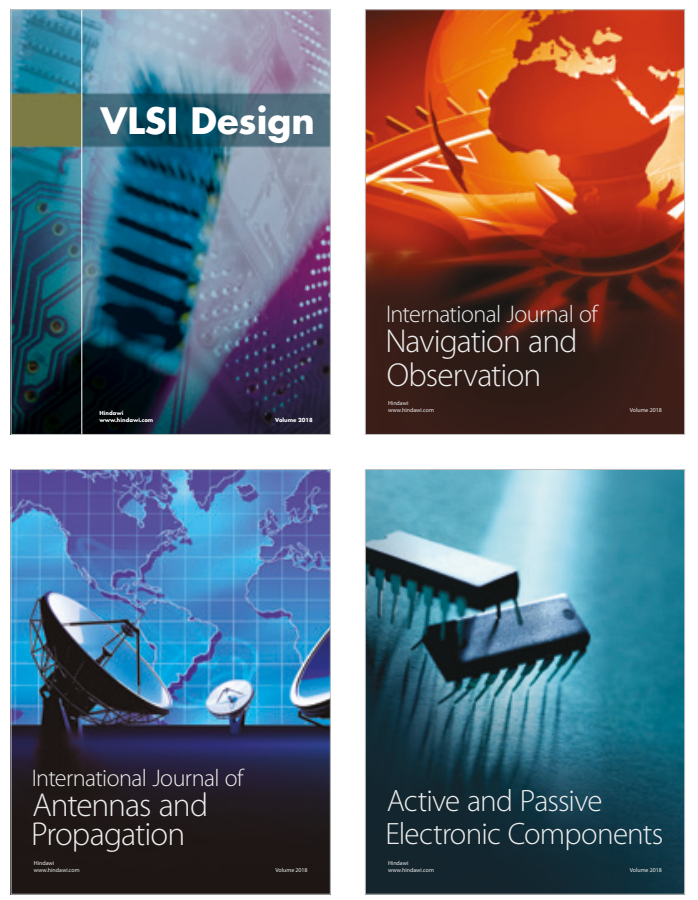
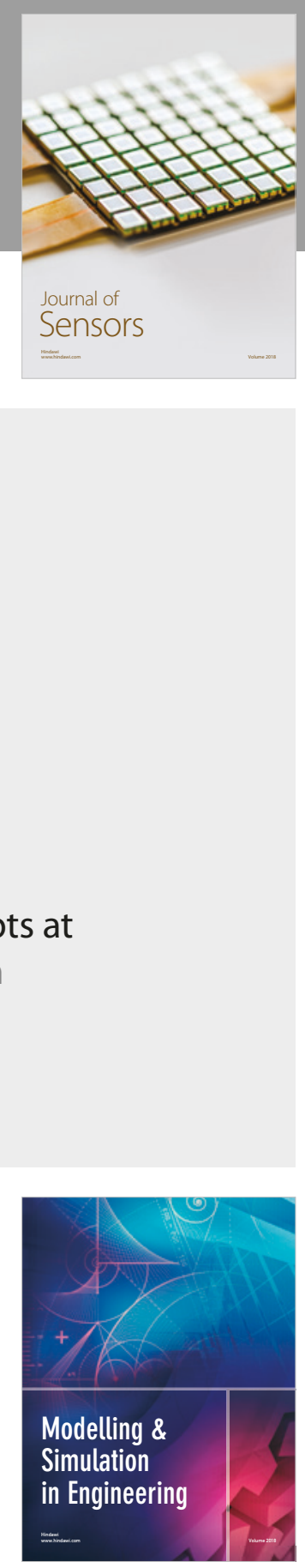

\section{Advances \\ Multimedia}
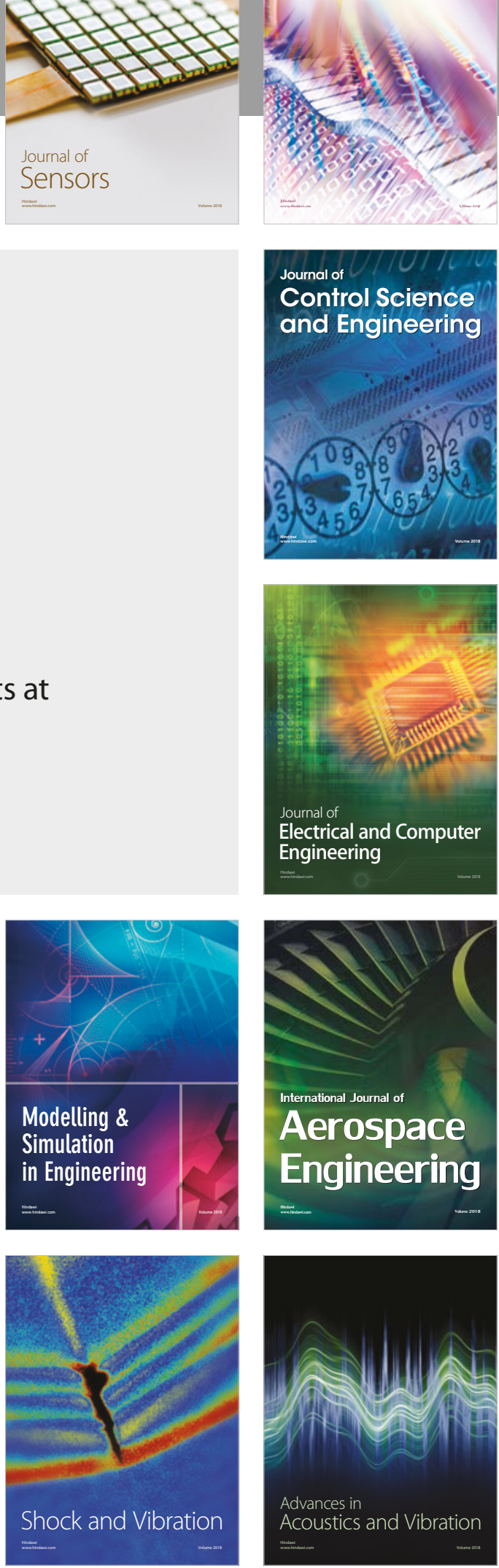